\title{
A Mathematical Bridge between Discretized Gauge Theories in Quantum Physics and Approximate Reasoning in Pairwise Comparisons
}

\author{
Jean-Pierre Magnot $\mathbb{i D}^{1,2}$ \\ ${ }^{1}$ LAREMA, UMR CNRS 6093, Université d'Angers, 2 bd Lavoisier, 49045 Angers Cedex 1, France \\ ${ }^{2}$ Lycée Jeanne d'Arc, 40 avenue de Grande Bretagne, 63000 Clermont-Ferrand, France \\ Correspondence should be addressed to Jean-Pierre Magnot; jp.magnot@gmail.com
}

Received 2 October 2017; Revised 16 December 2017; Accepted 26 December 2017; Published 23 January 2018

Academic Editor: Andrei D. Mironov

Copyright (c) 2018 Jean-Pierre Magnot. This is an open access article distributed under the Creative Commons Attribution License, which permits unrestricted use, distribution, and reproduction in any medium, provided the original work is properly cited.

We describe a mathematical link between aspects of information theory, called pairwise comparisons, and discretized gauge theories. The link is made by the notion of holonomy along the edges of a simplex. This correspondence leads to open questions in both fields.

\section{Introduction}

We present here an overview, addressed to physicists, of a possible bridge between gauge theories (and also some aspects of quantum gravity) with pairwise comparisons matrices and their applications in information theory and approximate reasoning. This is the reason why we rapidly summarize features in physics (assuming that they are known by the reader) and give more details for selected features on pairwise comparisons (PC) matrices (assuming that this field is less known). This paper is a companion work to [1-3] where, after a tentative in [4], the extension of the notion of classical PC matrices to matrices with coefficients in a group is considered, partially motivated (in my case) by the striking similarities with mathematical constructions in discretized gauge theories. A not complete list of reference about PC matrices is [5-7], oriented in our perspective, and a very partial list of references about gauge theories and their discretized forms is [8-19].

We begin with an oriented survey of selected problems in discretization of $G$-gauge theories, where $G$ is a Lie group, and a selection of features in evaluation of inconsistency in pairwise comparisons with coefficients in $\mathbb{R}_{+}^{*}$. Then we describe, following $[1,2]$, a straightforward extension of PC matrices with coefficients in $\mathbb{R}_{+}^{*}$ to a general Lie group $G$.
The link with gauge theories is performed via holonomy, which appears in discretizations described in $[3,16]$. In order to make a link with questions raised by computer scientists in private communications, we have to precise that any matrix group is a finite dimensional Lie group and that most finite dimensional groups can be expressed as matrix groups, for inversion and multiplication of matrices. We finish with the possible interpretations in both sides of this correspondence, first from quantities on PC matrices to gauge theories and secondly from second quantization to approximate reasoning.

\section{A Short and Not Complete Survey of Each Field of Knowledge}

We present here the two fields under consideration, discretized gauge theories and pairwise comparisons in approximate reasoning, in a way to highlight the correspondence.

2.1. Gauge Theories Discretized. The phase space of a (continuum) gauge theory is a space of connections on a (finite dimensional) principal bundle $P$ with structure group $G$ and with base $M$. We denote by $C(P)$ the space of connections considered. If $M$ is not compact and Riemannian, one often 
uses the space of connections which are smooth and squareintegrable. A gauge theory is defined by an action functional $S: C(P) \rightarrow \mathbb{R}$ which has to be minimized.

A discretized gauge theory is defined on a triangulation, a cubification or any other way to discretize the manifold $M$, and the principal bundle $P$ can be often trivial. Let us highlight two kind of discretizations:

(i) Whitney's discretization [20], where a connection $\theta \in C(P)$ is integrated on the 1-vertices of the chosen triangulation. This mimics a finite element method of approximation for scalar functions, and the discretized connection $\theta_{W}$ generates a $H^{1}$ approximation $\widetilde{\theta}$ of $\theta$, which $H^{1}$-converges to $\theta$ when refining the triangulation. The action functional $S$ is then evaluated on the finite dimensional space of connections $\widetilde{\theta}$ instead of the infinite dimensional space $C(P)$. This is, to our knowledge the most widely developed approach, but this approach seems to fail partially for nonabelian theories, partly because the trivialization is not gauge covariant. This leads to gauge-fixing strategies.

(ii) Holonomy discretization, mostly inspired by the ideas of quantum gravity [16], where connections along the edges are discretized through their holonomy. This approach requires mathematical precisions by fixing a preliminary gauge on the 1-vertices of the discretized manifold, but in a final analysis, only theories depending of secondary characteristic classes (e.g., Chern-Simons theory) can give rise to pathologies in gauge covariance, whereas gauge theories arising from primary characteristic classes (e.g., Yang-Mills theories) are fully gauge covariant when discretized [3].

2.2. Pairwise Comparisons, Consistency, and Inconsistency. A $n \times n$ pairwise comparisons (PC) matrix is a matrix

$$
A=\left(a_{i, j}\right)_{(i, j) \in \mathbb{N}_{n}^{2}}
$$

such that

$$
\begin{gathered}
\text { (i) } \forall(i, j) \in \mathbb{N}_{n}^{2}, a_{i, j} \in \mathbb{R}_{+}^{*} \text {, } \\
\text { (ii) } \forall i \in \mathbb{N}_{n}, a_{i, i}=1, \\
\text { (iii) } \forall(i, j) \in \mathbb{N}_{n}^{2}, a_{i, j}=a_{j, i}^{-1} .
\end{gathered}
$$

The coefficients $a_{i, j}$ are traditionally called ratios and are supposed to translate into a score the comparison of two states with numbers $i$ and $j$. When there exists a family of positive real numbers $\lambda_{1}, \ldots, \lambda_{n}$ such that

$$
a_{i, j}=\frac{\lambda_{i}}{\lambda_{j}}, \quad \forall(i, j) \in \mathbb{N}_{n}^{2},
$$

the PC matrix $A$ is called consistent. When it is not consistent, the formula

$$
a_{i, k} \cdot a_{k, j}=a_{i, j}
$$

does not hold for at least one choice of indexes $(i, j, k) \epsilon$ $\mathbb{N}_{n}^{3}$. By the way, it is easy to explain the inconsistency in PC matrices when we consider cycles of three comparisons, called triads and represented here as $(x, y, z) \in\left(\mathbb{R}_{+}^{*}\right)^{3}$, relative to the PC matrix

$$
\left(\begin{array}{ccc}
1 & x & y \\
x^{-1} & 1 & z \\
y^{-1} & z^{-1} & 1
\end{array}\right) \in M_{3}\left(\mathbb{R}_{+}^{*}\right)
$$

which do not have the "morphism of groupoid" property such as

$$
x \cdot z \neq y .
$$

The use of "inconsistency" has a meaning of a measure of inconsistency in this study, not the concept itself. In order to measure inconsistency, we define maps

$$
\text { ii: PC matrices } \longrightarrow \mathbb{R}_{+} \text {, }
$$

called inconsistency indicators, which are vanishing on consistent PC matrices and which intend to measure the lack of consistency, that is, intuitively speaking, the distance of a PC matrix to the nearest consistent PC matrix. In applications, PC matrices are modified with algorithms (which are often recursive) in order to minimize the chosen inconsistency indicator. One approach to inconsistency (originated in [6] and generalized in [5]) can be reduced to a simple observation:

(i) Search all triads (which generate all 3 by 3 PC submatrices) and locate the worst triad with an inconsistency indicator (ii).

(ii) The inconsistency indicator evaluated on the worst triad becomes the inconsistency indicator evaluated on the entire PC matrix.

Expressing it a bit more formally in terms of triads (the upper triangle of a PC submatrix $3 \times 3$ ), we have

$$
\mathrm{ii}_{3}(x, y, z)=1-\min \left\{\frac{y}{x z}, \frac{x z}{y}\right\}=1-e^{-|\ln (y / x z)|} .
$$

The expression $|\ln (y / x z)|$ stands for the distance of the triad $T$ to the "nearest" consistent PC matrix. When this distance increases, the $\mathrm{ii}(x, y, z)$ also increases. It is important to notice here that this definition allows us to localize the inconsistency in the PC matrix, which is of importance for most applications. For higher rank matrices, $\mathrm{ii}_{3}$ is evaluated on each $3 \times 3$ matrix, taking the supremum of the obtained values. 
Another possible definition of the inconsistency indicator can also be defined (following [7]) as

$$
\mathrm{ii}_{n}(A)=1-\min _{1 \leq i<j \leq n} \min \left(\frac{a_{i j}}{a_{i, i+1} a_{i+1, i+2} \cdots a_{j-1, j}}, \frac{a_{i, i+1} a_{i+1, i+2} \cdots a_{j-1, j}}{a_{i j}}\right)
$$

since the matrix $A$ is consistent if and only if for any $1 \leq i<$ $j \leq n$ the following equation holds:

$$
a_{i j}=a_{i, i+1} a_{i+1, i+2} \cdots a_{j-1, j} .
$$

This is equivalent to

$$
\mathrm{ii}_{n}(A)=1-\max _{1 \leq i<j \leq n}\left(1-e^{-\left|\ln \left(a_{i j} / a_{i, i+1} a_{i+1, i+2} \cdots a_{j-1, j}\right)\right|}\right) .
$$

One of the main features in applications is to minimize the inconsistency indicator ii. This is the reason why, instead of using the inconsistency indicator ii defined before, there is plethora of inconsistency indicators. Each inconsistency indicator intends to measure how far a PC matrix is from the set of consistent $\mathrm{PC}$ matrices, which, for $3 \times 3 \mathrm{PC}$ matrices, is a 2-dimensional manifold of matrices of the form

$$
\left(\begin{array}{ccc}
1 & x & x y \\
x^{-1} & 1 & y \\
x^{-1} y^{-1} & y^{-1} & 1
\end{array}\right), \quad \text { with }(x, y) \in\left(\mathbb{R}_{+}^{*}\right)^{2}
$$

Unfortunately, the notion and the theory of inconsistency indicators are not actually fixed and achieved, and many competing, incompatible approaches are actually developed. In order to relate PC matrices to gauge theories, we need to generalize (quite straightway) the framework described before, changing the group $\mathbb{R}_{+}^{*}$ to any Lie group $G$. In the setting of gauge theories, $G$ is in most cases a finite dimensional, compact (or at least unimodular) group which can be represented as a matrix group. But this assumption is not technically necessary for the generalization and will be only recovered when discussing the possible correspondences announced.

\section{The Matrix of Holonomies and Pairwise Comparisons}

We first follow [2]. Let $I$ be a set of indexes among $\mathbb{Z}, \mathbb{N}$, or $\{0, \ldots, n\}$ for some $n \in \mathbb{N}^{*}$.

Definition 1. Let $(G, \cdot)$ be a group. A PC matrix is a matrix

$$
A=\left(a_{i, j}\right)_{(i, j) \in I^{2}}
$$

such that

(1) $\forall(i, j) \in I^{2}, a_{i, j} \in G$,

(2) $\forall(i, j) \in I^{2}, a_{j, i}=a_{i, j}^{-1}$, where -1 is the inversion in $G$,

(3) $a_{i, i}=1_{G}$.
The matrix $A$ is covariantly consistent if

$$
a_{i, j} \cdot a_{j, k}=a_{i, k}, \quad \forall(i, j, k) \in I^{3} .
$$

Due to the contravariant composition thereafter, we will use the following notion: the PC matrix is contravariantly consistent if

$$
a_{j, k} \cdot a_{i, j}=a_{i, k}, \quad \forall(i, j, k) \in I^{3} .
$$

These two notions are dual and depend on which order we require for the group multiplication. Similarly, a contravariant consistent PC matrix $A=\left(a_{i, j}\right)_{(i, j) \in I^{2}}$ generates a covariant consistent PC matrix $B=\left(b_{i, j}\right)_{(i, j) \in I^{2}}$ setting

$$
b_{i, j}=a_{i, j}^{-1}=a_{j, i} \quad \text { in } G \text {. }
$$

For convenience, we use the term covariant PC matrix (resp., contravariant PC matrix) when covariant consistency (resp., contravariant consistency) is naturally required. In order to make the difference more clear with the more general cases described in next section, we have to recall that each coefficient here is in a group, and hence there is no vanishing coefficient in the matrix $A$.

\section{Theorem 2.}

$$
A \text { is consistent } \Longleftrightarrow a_{i, j}=\lambda_{i}^{-1} \cdot \lambda_{j}, \quad \exists\left(\lambda_{i}\right)_{i \in \mathbb{N}_{n}} .
$$

Let $n \in \mathbb{N}^{*}$ and

$$
\begin{aligned}
& \Delta_{n}=\left\{\left(x_{0}, \ldots, x_{n}\right) \in \mathbb{R}^{n+1} \mid\left(\sum_{i=0}^{n} x_{i}=1\right)\right. \\
& \left.\wedge\left(\forall i \in\{0, \ldots, n\}, x_{i} \geq 0\right)\right\}
\end{aligned}
$$

be an $n$-simplex. This simplex can be generalized to the infinite dimension:

$$
\begin{aligned}
& \Delta_{\mathbb{N}}=\left\{\left(x_{n}\right)_{n \in \mathbb{N}} \in l^{1}\left(\mathbb{N}, \mathbb{R}_{+}^{*}\right) \mid \sum_{i=0}^{\infty} x_{i}=1\right\}, \\
& \Delta_{\mathbb{Z}}=\left\{\left(x_{n}\right)_{n \in \mathbb{Z}} \in l^{1}\left(\mathbb{Z}, \mathbb{R}_{+}^{*}\right) \mid \sum_{i \in \mathbb{Z}} x_{i}=1\right\},
\end{aligned}
$$

where the summation over $\mathbb{Z}$ is done by integration with respect to the counting measure. In the sequel, $\Delta$ will denote $\Delta_{n}, \Delta_{\mathbb{N}}$, or $\Delta_{\mathbb{Z}}$. Let $\left(s_{i}\right)$ be the 0 -vertices of $\Delta$. Since $\Delta$ is smoothly contractible, any $G$-principal bundle over $\Delta$ is 
TABLE 1

\begin{tabular}{lcc}
\hline PC matrices & Discretized gauge theories & Continuum gauge theories \\
\hline Consistency & 0 -holonomy & 0 -curvature \\
Consistency in $3 \times 3$ matrices & 0 -holonomy on the border of a 2-simplex $\Delta_{2}$ & $\Omega(X, Y)=0$ with $X, Y$ tangent to $\Delta_{2}$ \\
Koczkodaj's inconsistency indicator ii & $\begin{array}{c}\sup d\left(\operatorname{Hol}\left(\partial \Delta_{2}\right), 1_{G}\right) \text { when } \Delta_{2} \text { is any 2-simplex of the } \\
\text { triangulation }\end{array}$ & sup $\left\|_{M}\right\| \Omega \|$ \\
Minimization of inconsistency & $?$ & Minimization of the curvature norm \\
\hline
\end{tabular}

isomorphic to $\Delta \times G$ and a $G$-connection 1 -form on $\Delta$ is a 1-form $\theta \in \Omega^{1}(\Delta, \mathfrak{g})$, which extends to a $G$-covariant 1-form in $\Omega^{1}(\Delta, \mathfrak{g})$, with respect to the coadjoint action of $G$ on $\mathfrak{g}$. We define a gauge $\left(g_{i}\right)_{i \in I} \in G^{I}$ with $\widetilde{\gamma_{i}}(1)=\left(\gamma_{i}(1), g_{i}\right)$ where the piecewise smooth paths $\gamma_{i}$ are defined by the standard composition $*$ of the paths along 1 -vertices $\left[s_{j}, s_{j+1}\right]$ of $\Delta$ setting:

$$
\begin{array}{ll}
\gamma_{i}=\left[s_{0}, s_{1}\right] * \cdots *\left[s_{i-1}, s_{i}\right] & \text { if } i>0, \\
\gamma_{i}=\left[s_{0}, s_{-1}\right] * \cdots *\left[s_{i+1}, s_{i}\right] & \text { if } i<0 .
\end{array}
$$

Let Hol the (mathematical) holonomy on loops relative to a fixed connection $\theta$. We set $g_{i}=\operatorname{Hol}_{\left(s_{0}, 1_{G}\right)} \gamma_{i}$. Let us recall that, for two paths $c$ and $c^{\prime}$ such that $c * c^{\prime}$ exists (i.e., $c(1)=c^{\prime}(0)$ ), if $p=\left(c(0), e_{G}\right), p^{\prime}=\left(c^{\prime}(0), e_{G}\right)$, and $h=\operatorname{Hol}_{p} c$, we have

$$
\begin{aligned}
\operatorname{Hol}_{p}\left(c * c^{\prime}\right) & =\operatorname{Hol}_{p}(c) \cdot\left(h^{-1} \operatorname{Hol}_{p^{\prime}}\left(c^{\prime}\right) h\right) \\
& =\operatorname{Hol}_{p^{\prime}}\left(c^{\prime}\right) \cdot \operatorname{Hol}_{p}(c) .
\end{aligned}
$$

This enables the (physics) definition of holonomy present, for example, in [16] that we apply to the edges $\left[s_{i}, s_{j}\right]$ and identify with the coefficients $a_{i, j}$ :

$$
a_{i, j}=g_{j} \cdot \operatorname{Hol}_{\left(s_{0} ; e_{G}\right)}\left(\gamma_{i}\left[s_{i}, s_{j}\right] \gamma_{j}^{-1}\right) \cdot g_{i}^{-1} .
$$

In the light of these specifications, we set, for any connection $\theta \in \Omega^{1}(\Delta, \mathfrak{g})$,

$$
A=\operatorname{Mat}\left(a_{i, j}\right)
$$

and the required notion of consistency is contravariant consistency.

Proposition 3. A is a PC matrix with coefficients in $G$.

Proof. This follows from holonomy in "reverse orientation."

Let $\gamma_{i, j, k}=\gamma_{i} *\left[s_{i}, s_{j}\right] *\left[s_{j}, s_{k}\right] *\left[s_{k}, s_{i}\right] * \gamma_{i}^{-1}$ be the loop based on $s_{i}$ along the border of the oriented 2-vertex $\left[s_{i}, s_{j}, s_{k}\right]$, where $*$ is the composition of paths.

Contravariant consistency seems to fit naturally with flatness of connections:

$$
\begin{aligned}
a_{i, k} & =a_{j, k} \cdot a_{i, j} \\
a_{k, i} \cdot a_{j, k} \cdot a_{i, j} & =a_{i, i}=1_{G} \Longleftrightarrow \\
\operatorname{Hol}\left(\gamma_{i, j, k}\right) & =1_{G},
\end{aligned}
$$

By fixing an indicator map, defined in [4] as

$$
\text { In }: G \longrightarrow \mathbb{R}_{+}
$$

to $\operatorname{In}\left(1_{G}\right)=0$, we get a generalization of the inconsistency indicator by setting

$$
\mathrm{ii}_{\text {In }}=\sup \left\{\operatorname{In}\left(\operatorname{Hol}\left(\gamma_{i, j, k}\right)\right) \mid(i, j, k) \in I^{3}\right\} .
$$

For example, if $d$ is a left-invariant distance on $G$, a natural indicator map can be

$$
\text { In }: g \longmapsto d\left(1_{G}, g^{-1}\right)
$$

Such definitions extend to triangularized manifolds along the lines of [3], where one can see that the notion of holonomy on a manifold can be discretized, inserting "gaps" (i.e., 0 entries) on a larger matrix gathering all the PC matrices over the simplexes of the triangulation, along the lines of [2]. We then recover the discretization of connections via holonomies described in [16]. When $G=\mathbb{R}_{+}^{*}$ In (classical) pairwise comparisons, matrix coefficients are scalar, which enable simplifying the settings obtained. The indicator $\mathrm{ii}_{n}$ appears as the distance of the holonomy of the loop $\left[s_{i} s_{i+1} \cdots s_{i+n} s_{i}\right]$ to identity.

\section{Correspondences and Open Questions}

We give the table of correspondences (see Table 1) that we comment on. We first highlight how the notions on PC matrices correspond to geometric objects.

Minimization of inconsistency is an important feature for applications of PC matrices. For decision making, it consists in adapting slightly the parameters of the studied situation in order to make "approximately consistent" choices. In order to check if the adapted PC matrix is "approximately consistent," the criteria are given by the chosen inconsistency indicator, for example, Koczkodaj's ii, which needs to have a value $\epsilon \geq 0$ small enough. Again for Koczkodajs ii, it is trivial to see that the sets

$$
V_{\epsilon}=\mathrm{ii}_{3}^{-1}[0 ; \epsilon[
$$

form a filter base of open neighborhoods of the set of consistent PC matrices. The analog for continuum gauge theories is considering

$$
U_{\epsilon}=\left\{\theta \in C(P) \mid \sup _{M}\|\Omega\|<\epsilon\right\} .
$$


TABLE 2

\begin{tabular}{|c|c|c|}
\hline Continuum integration & Discretized integration & PC matrices \\
\hline Heuristic Lebesgue measure & Lebesgue measure (finite dimension, Whitney discretization) & $?$ \\
\hline Integral to be studied & $\begin{array}{c}\text { Product measure on } G^{n(n-1) / 2} \text { ( } G \text { compact), on the } \\
\text { discretization by holonomies }\end{array}$ & $\begin{array}{l}\text { Measures on } n \times n \mathrm{PC} \\
\text { matrices }\end{array}$ \\
\hline
\end{tabular}

This defines a filter base of open neighborhoods of the set of 0 -curvature connections. This leads to the following open problem.

On one hand, the procedures developed to get consistencizations of PC matrices are specific to the case $G=$ $\mathbb{R}_{+}^{*}$. However, there exist many methods for minimizing functional, and one may wonder whether minimization of the curvature norm has a physical meaning. The corresponding physical quantity would be the Yang-Mills action functional, but we get here an average value instead of a supremum. This questions the choice of the supremum in the formula

$$
\begin{aligned}
\mathrm{ii}_{3} & (A) \\
& =\sup \left\{\mathrm{ii}_{3}(B) \mid B \text { is a } 3 \times 3 \text { PC submatrix of } A\right\} .
\end{aligned}
$$

On the other hand, considering flat connections, or only connections such that $\Omega=d \theta$ is a common feature in gauge fixing. Since minimal states represent stable solutions, consistencization may appear as a way to equilibrium.

Let us now reverse the perspective and consider second quantization. We now analyze how the Feynman-like integration (i.e., cylindrical integration for discretized theories) may arise in PC matrices (see Table 2).

If $G$ is compact, we can assume that it is of volume 1 . In this case, there is a convergence at the continuum limit to an integral. This is the approach suggested in [16], which is an alternative approach to the classical integration with respect to the heuristic Lebesgue integral (see, e.g., [10]). On one hand, for both cases, the possible interpretations in terms of applications of PC matrices are not investigated. Heuristically, measures on PC matrices may arise when evaluations are random, or subject to measurement errors. On the other hand, PC matrices may furnish an interpretation in terms of information theory of Feynman type integration.

\section{Conflicts of Interest}

The author declares that there are no conflicts of interest with respect to the publication of this paper.

\section{References}

[1] W. W. Koczkodaj and J-P. Magnot, https://pdfs.semanticscholar .org/4c22/f469004fd0e03172e661c1611945eb3348a6.pdf.

[2] J.-P. Magnot, "On pairwise comparisons with values in a group: algebraic structures," 2016, https://arxiv.org/abs/1608.07468.

[3] J.-P. Magnot, Remarks on a new possible discretization scheme for gauge theories, https://arxiv.org/abs/1706.09518.

[4] W. W. Koczkodaj, J. Szybowski, and E. Wajch, "Inconsistency indicator maps on groups for pairwise comparisons," International Journal of Approximate Reasoning, vol. 69, pp. 81-90, 2016.
[5] Z. Duszak and W. W. Koczkodaj, "Generalization of a new definition of consistency for pairwise comparisons," Information Processing Letters, vol. 52, no. 5, pp. 273-276, 1994.

[6] W. W. Koczkodaj, "A new definition of consistency of pairwise comparisons," Mathematical and Computer Modelling, vol. 18, no. 7, pp. 79-84, 1993.

[7] W. W. Koczkodaj and R. Szwarc, "On axiomatization of inconsistency indicators for pairwise comparisons," Fundamenta Informaticae, vol. 132, no. 4, pp. 485-500, 2014.

[8] S. Albeverio and R. Høegh-Krohn, "Brownian Motion, Markov Cosurfaces, Higgs Fields," in Fundamental Aspects of Quantum Theory, vol. 144 of NATO ASI Series, pp. 95-104, Springer US, Boston, MA, 1986.

[9] S. Albeverio, R. Hoegh-Krohn, and H. Holden, "Markov cosurfaces and gauge fields," Acta Physica Austriaca, no. Supplement 26, pp. 211-231, 1984.

[10] S. A. Albeverio, R. J. Høegh-Krohn, and S. Mazzuchi, Mathematical Theory of Feynman Path Integrals; An Introduction, vol. 523 of Lecture Notes in Mathematics 523, Springer, 2005.

[11] S. Albeverio and B. Zegarlinski, "Construction of convergent simplicial approximations of quantum fields on Riemannian manifolds," Communications in Mathematical Physics, vol. 132, no. 1, pp. 39-71, 1990.

[12] A. Hahn, "The Wilson loop observables of Chern-Simons theory on $\mathbb{R} 3$ in axial gauge," Communications in Mathematical Physics, vol. 248, no. 3, pp. 467-499, 2004.

[13] M. Q. Huber, D. R. Campagnari, and H. Reinhardt, "Vertex functions of Coulomb gauge Yang-Mills theory," Physical Review D: Particles, Fields, Gravitation and Cosmology, vol. 91, article 025014, no. 2, 2015.

[14] A. P. Lim, "Non-abelian gauge theory for Chern-Simons path i ntegral on R3," Journal of Knot Theory and Its Ramifications, vol. 21, article 1250039, no. 04, 2012.

[15] H. Reinhardt, "Yang-Mills in axial gauge," Physical Review, vol. 55, pp. 2331-2346, 1997.

[16] C. Rovelli and F. Vidotto, Covariant Loop Quantum Gravity, Cambridge University Press, Cambridge, UK, 2014.

[17] S. Sen, S. Sen, J. C. Sexton, and D. H. Adams, "Geometric discretization scheme applied to the Abelian Chern-Simons theory," Physical Review E: Statistical, Nonlinear, and Soft Matter Physics, vol. 61, no. 3, pp. 3174-3185, 2000.

[18] A. N. Sengupta, "Yang-Mills in two dimensions and ChernSimons in three," in Chern-Simons Theory: 20 years after, J. Anderson, H. U. Boden, A. Hahn, and B. Himpel, Eds., pp. 311320, AMS/IP Studies in Advanced Mathematics, 2011.

[19] A. N. Sengupta, "Connections over two-dimensional cell complexes," Reviews in Mathematical Physics, vol. 16, no. 3, pp. 331352, 2004.

[20] H. Whitney, Geometric Integration Theory, Princeton University Press, Princeton, NJ, USA, 1957. 


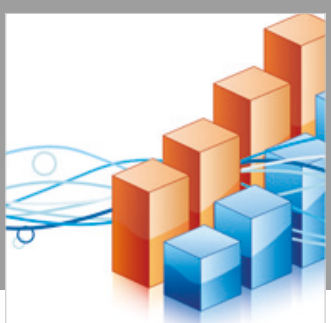

Advances in

Operations Research

\section{-n-m}
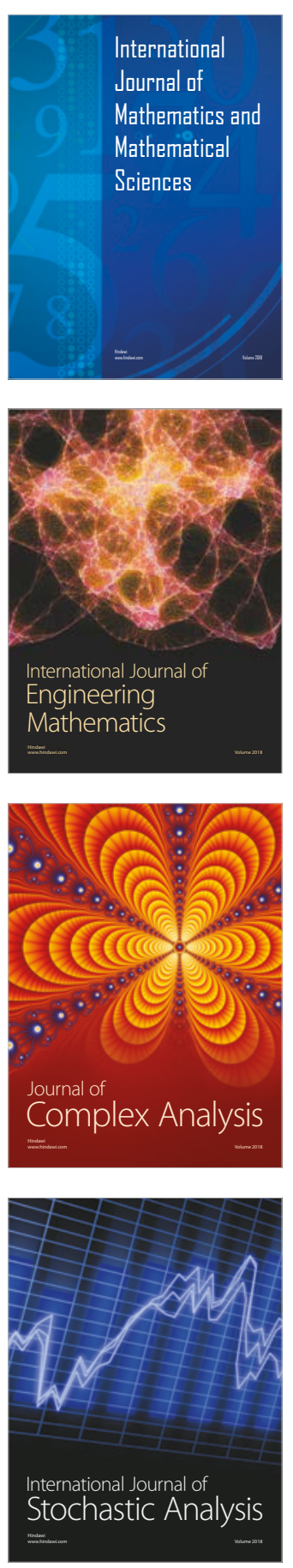
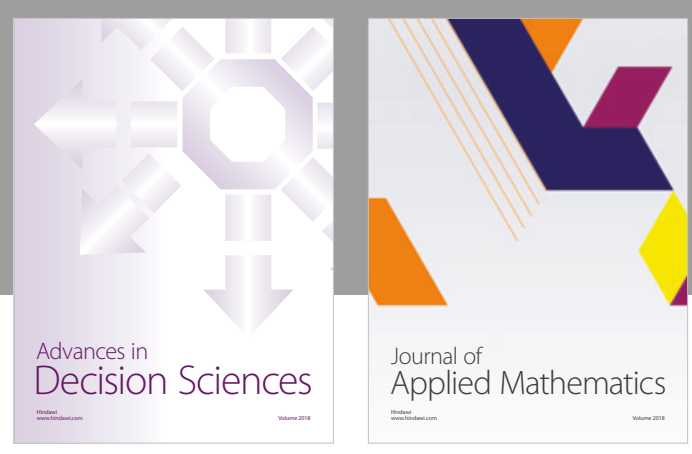

Journal of

Applied Mathematics
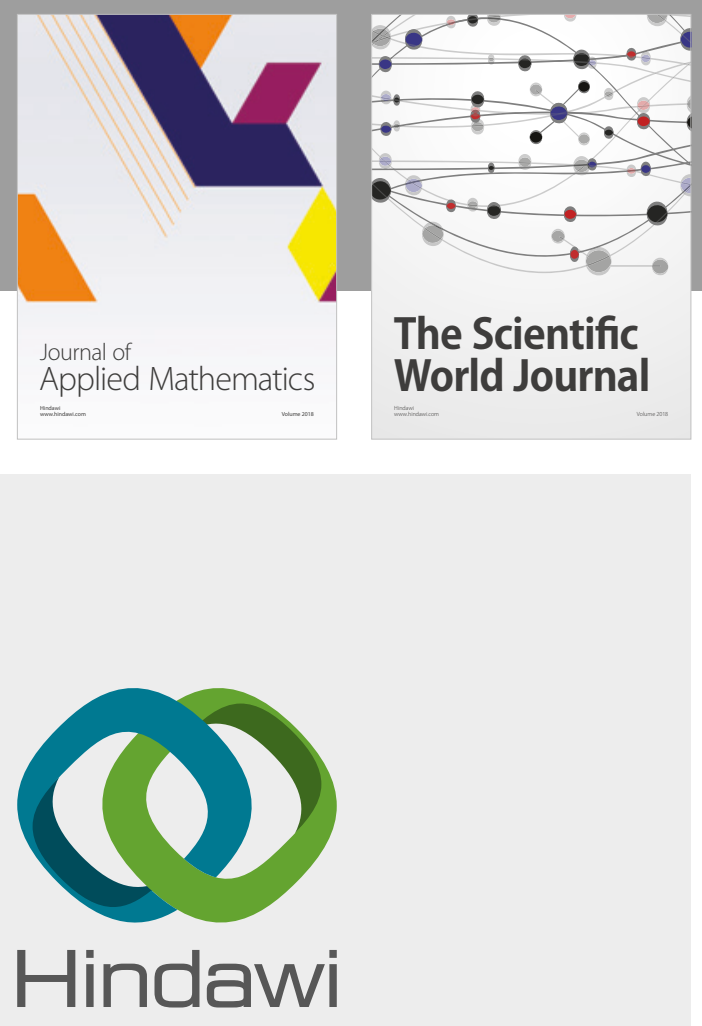

Submit your manuscripts at

www.hindawi.com

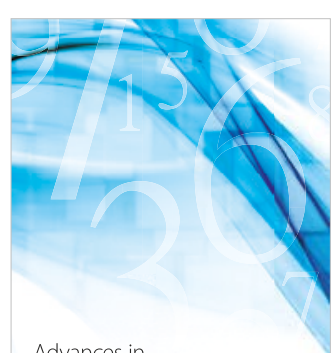

Advances in
Numerical Analysis
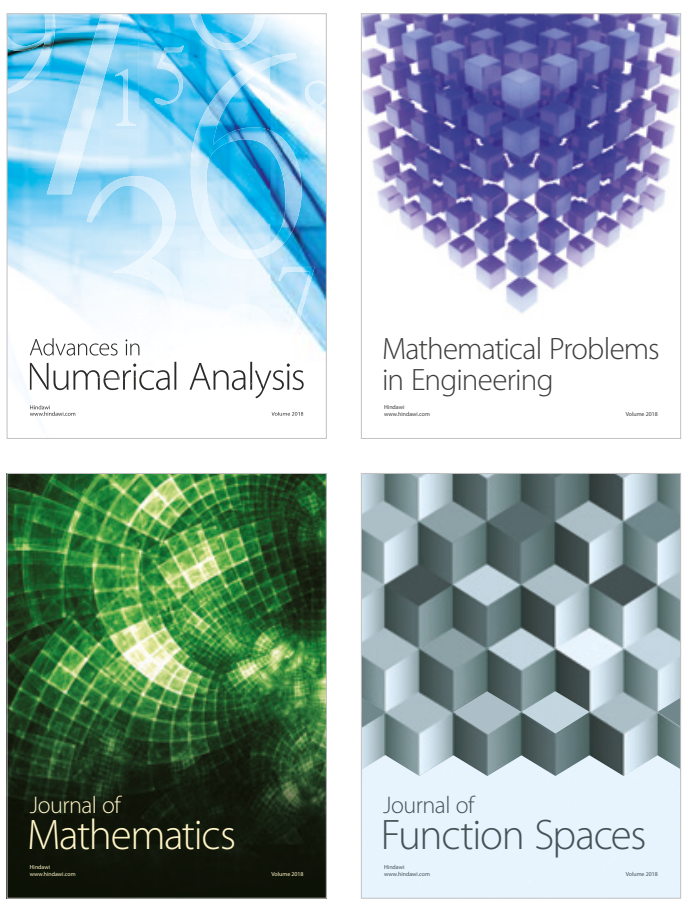

Mathematical Problems in Engineering

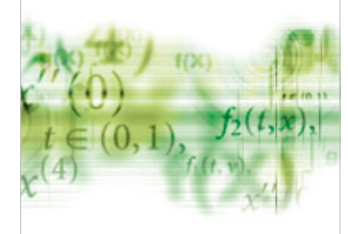

International Journal of

Differential Equations

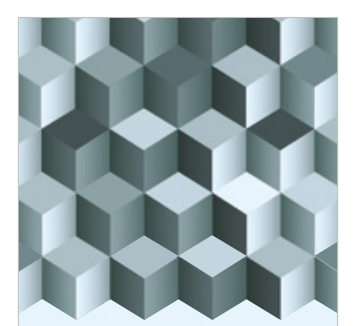

Journal of

Function Spaces

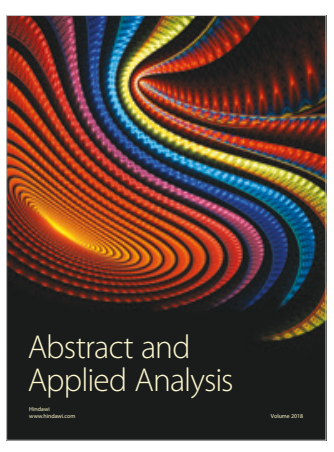

The Scientific

World Journal

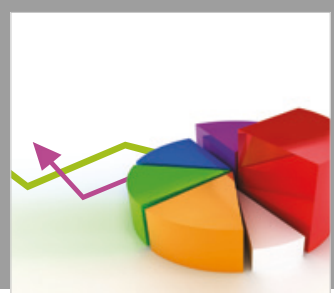

Journal of

Probability and Statistics
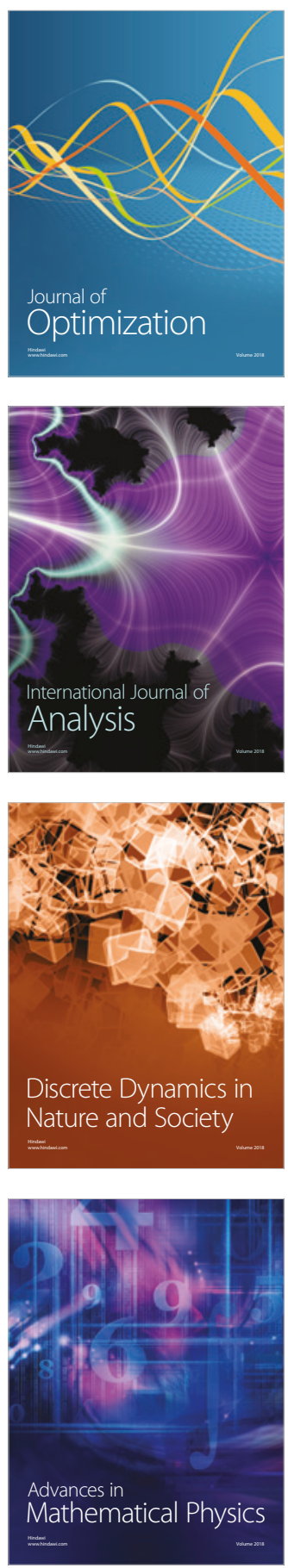